\title{
Accuracy and Safety of Distal Femoral Valgus Correction: A Comparison of Three Techniques
}

\author{
Christopher lobst ${ }^{1}$, Mohammed Waseemuddin², Anirejuoritse Bafor ${ }^{3}$
}

\begin{abstract}
Introduction: There are several methods for correcting distal femoral valgus deformity in skeletally mature patients including fixator-assisted plating (FAP), fixator-assisted nailing (FAN) and nailing using the reverse planning method. The fixator-assisted techniques have been previously compared in the literature and found to be similarly accurate. This study is the first to compare all three procedures in a single series.

Materials and methods: A retrospective review of patients who had undergone distal femoral valgus correction at a single institution between March 2017 and February 2020 was undertaken. Three different patient groups were identified based on the surgical technique used: the FAP, the FAN and the reverse planning method. The mechanical lateral distal fimoral angle (mLDFA) was recorded and compared preoperatively and postoperatively. The body mass index (BMI), duration of surgery, postoperative range of motion (ROM) and complication profile for each patient were also recorded and compared.

Results: A total of 27 limbs in 24 patients were included in this study. There were 8 male and 16 female patients. There were 10 limbs from 9 patients in the reverse planning group, 11 limbs from 11 patients in the FAN group and 6 limbs from 4 patients in the FAP group. There was a statistically significant difference in the mean preoperative and postoperative mLDFA for each of the individual groups $(p<0.0001$ for each group). All patients had restoration of the mLDFA to within normal limits except one patient in the reverse planning group. This was purposefully performed to compensate for an ipsilateral proximal tibial deformity. There was no statistically significant difference in the mean preoperative and postoperative mLDFA across the groups ( $p=0.2897$ and 0.3440 , respectively). The operative time of the reverse planning method and the FAP were significantly shorter than FAN ( $p=0.0016$ and $p=0.0035$, respectively). The mean final knee ROM amongst the groups was similar $(p=0.8190)$. We recorded no infections or union complications in any group. There was one case of hardware irritation causing lateral knee pain that did not require treatment in the reverse planning group and one fracture through a temporary half-pin site in the FAN group. All six plates in the FAP group had to be removed following union on account of localised discomfort from the hardware.

Conclusion: The reverse planning method, the FAN and the FAP are comparable in terms of accuracy in achieving correction of distal femur valgus deformity in skeletally mature patients. The reverse planning method had the best combination of outcomes in this study since it was just as accurate as the FAN and the FAP techniques and did not require any additional surgeries. While both the reverse planning and the FAP were faster than the FAN technique, the reverse planning method allows the ability to perform both deformity correction and lengthening. Ultimately, the decision of which technique to use depends on a combination of the patient's preference and the surgeon's level of comfort with the technique.

Keywords: Fixator-assisted nailing, Fixator-assisted plating, Reverse planning.

Strategies in Trauma and Limb Reconstruction (2020): 10.5005/jp-journals-10080-1455
\end{abstract}

\section{INTRODUCTION}

Angular deformities around the knee joint can lead to pain, decreased function and, potentially, osteoarthritis. ${ }^{1}$ Lower extremity varus or valgus deformities are also often cosmetically unappealing to the patient and can make future joint reconstruction more difficult if not corrected. ${ }^{2}$ The deformity can originate in the femur, tibia or a combination of both sites. While there are multiple possible aetiologies for the deformity, the goal in each case is to restore the mechanical axis alignment of the lower extremity.

Several different methods have been described to correct angular deformities using closing or opening wedge osteotomies. Definitive fixation of the femur with an external fixator has been shown to be accurate and effective. ${ }^{3}$ However, it is also known to be cumbersome and uncomfortable for the patient, especially when pin site infections occur. Internal fixation techniques avoid pin site issues but require larger incisions and soft tissue dissection. ${ }^{4}$ In addition, once the internal fixation is placed, the amount of correction is not adjustable postoperatively without a return to the operating room. Recently, hybrid techniques, such as fixator-assisted nailing (FAN) and fixator-assisted plating (FAP), have become popular. ${ }^{5-14}$ These methods combine the flexibility of external fixation with the
${ }^{1-3}$ Department of Orthopedic Surgery, Nationwide Children's Hospital, Columbus, Ohio, USA

Corresponding Author: Christopher lobst, Department of Orthopedic Surgery, Nationwide Children's Hospital, Columbus, Ohio, USA, Phone: +1 (614) 722-5175, e-mail: Christopher.lobst@nationwidechildrens.org How to cite this article: lobst $C$, Waseemuddin M, Bafor A. Accuracy and Safety of Distal Femoral Valgus Correction: A Comparison of Three Techniques. Strategies Trauma Limb Reconstr 2020;15(1):41-46.

Source of support: Nil

Conflict of interest: Dr lobst is a consultant for Orthofix, Smith and Nephew, and NuVasive. Dr Waseemuddin and Dr Bafor have no conflicts of interest to report

convenience of internal fixation. Previous studies comparing the FAN technique and the FAP technique have demonstrated comparable accuracy of correction and operative times..$^{15,16}$

The reverse planning technique introduced by Baumgart in 2009 is an alternative technique for correcting deformity around the knee with an intramedullary nail. ${ }^{17}$ In reverse planning, the surgeon first determines the desired final position of the bone and then works backwards to the existing deformity. Meticulous preoperative 
planning creates an illustration of the desired correction and the nail placement that the surgeon uses intraoperatively as a guide. Rigid reamers and blocking screws are used rather than an external fixator to control and maintain the amount of correction intraoperatively. By avoiding the use of an external fixator, this method simplifies the amount of equipment necessary for the case and eliminates any additional patient morbidity from the placement of the temporary half pin. Reverse planning has been shown to have a high level of accuracy in the correction of lower extremity deformity. ${ }^{17-19}$ The goal of this study is to compare the accuracy of the reverse planning technique in restoring the mechanical lateral distal femoral angle ( $m L D F A)$ with the FAN and the FAP techniques in patients with distal femoral valgus deformity. This analysis will also compare the operative time and the complication rate of the three techniques.

\section{Materials and Methods}

After obtaining institutional review board approval, a retrospective review between March 2017 and February 2020 of a single surgeons' experience correcting distal femoral valgus deformity was performed. Three different techniques were utilised to correct the distal femoral valgus deformity: FAP, FAN and reverse planning. The decision to perform either plating or intramedullary nailing was based on the patient's preference. For the intramedullary nail cases, FAN was used prior to the senior author starting to use the reverse planning technique. Since 2019 , reverse planning has been used in all nail cases. This series includes the surgeon's initial cases with the reverse planning method factoring in the learning curve. Fixator-assisted plating was only offered to patients as an option if they did not require lengthening. A brief description of each surgical technique is provided.

\section{Fixator-assisted Plating Technique (Fig. 1)}

Preoperative surgical planning was performed using Paley's principles of deformity analysis. ${ }^{20}$ The level of the osteotomy and the size of the plate to be used were also determined preoperatively. A small incision was made distally, and the plate was inserted from distal to proximal in a submuscular fashion. The location of the osteotomy relative to the plate screw hole distribution was identified and drill holes in the femur were made at this level. Two $6 \mathrm{~mm}$ half pins were applied in parallel from the medial side of the femur with one distal and one proximal to the proposed osteotomy site. Each half pin was placed orthogonal to its respective bone segment. The osteotomy was completed with an osteotome. The required rotation, translation and angulation were performed as per the preoperative plan. The corrected position was held by the fixator. The locking plate was reinserted in a submuscular fashion and verified to be in the appropriate position in both the coronal and the sagittal planes. Distally, the plate was initially fixed with a non-locking screw to pull the plate to the bone. Additional locking screws were then added. Proximally, the plate sits off the bone and is only fixed with locking screws. Once stable fixation was achieved, the fixator was removed. Restoration of the mechanical axis was verified using an intraoperative grid.

\section{Fixator-assisted Nailing Technique (Fig. 2)}

Preoperative surgical planning was performed using Paley's principles of deformity analysis. ${ }^{20}$ The level of the osteotomy, the nail length and the nail diameter to be used were also determined preoperatively. Multiple percutaneous drill holes were made at the distal femoral metaphyseal osteotomy site. Two $6 \mathrm{~mm}$ half pins were placed percutaneously and parallel with one distal and one proximal to the osteotomy site. Both pins were placed from the lateral side orthogonal to the axis of the femur and posterior to the path of the nail. The percutaneous osteotomy was completed with an osteotome. The desired rotation, translation and angulation were performed and held in position with the fixator. A medial parapatellar approach was used to insert the nail guide wire followed by reaming. After the nail was inserted and locked, blocking screws
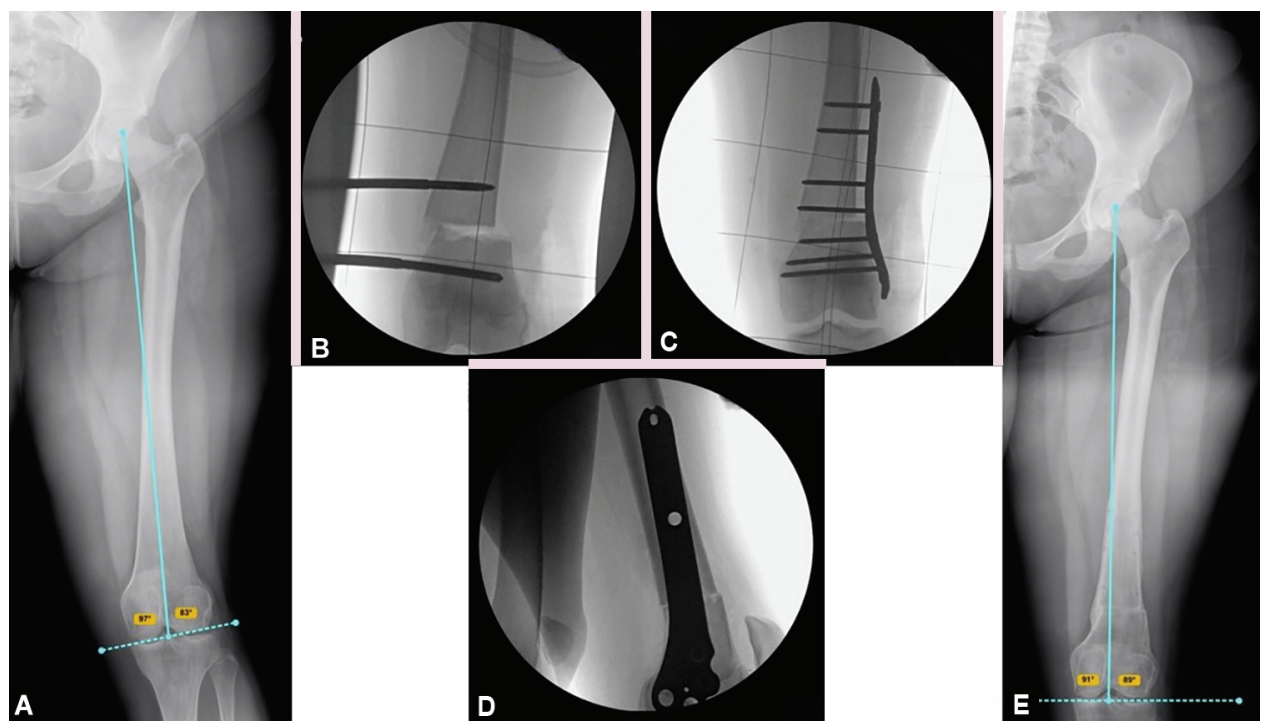

Figs $1 \mathrm{~A}$ to E: Fixator-assisted plating technique: (A) Preoperative anteroposterior femur radiograph in a patient with a history of recurrent patellar subluxation and a mechanical lateral distal femoral angle (mLDFA) measuring $83^{\circ}$; (B) Intraoperative image demonstrating half pins placed from the medial side of the femur above and below the osteotomy holding the acute correction in place via a pin-to-bar construct; (C) Intraoperative anteroposterior image demonstrating the final plate placement after fixator removal. The proximal portion of the plate is sitting off of the bone and is fixed with only locking screws; (D) Intraoperative lateral image demonstrating the osteotomy and plate alignment in the sagittal plane; (E) Anteroposterior femoral radiograph demonstrating the final result after union of osteotomy and plate removal. Patient has normal mLDFA 
were placed to maintain the alignment prior to removal of the fixator. Restoration of the mechanical axis was verified using an intraoperative grid.
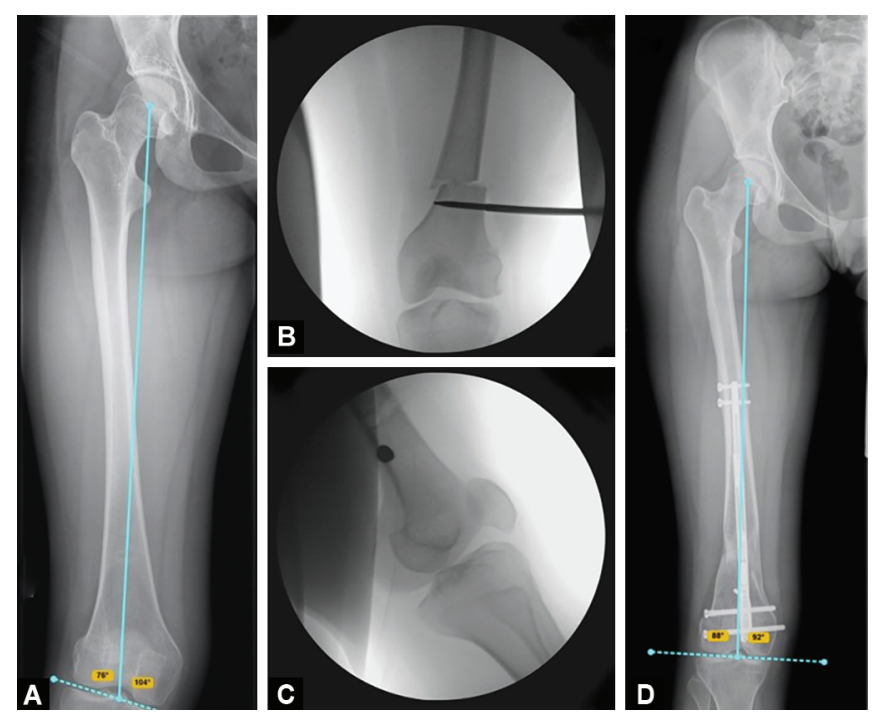

Figs 2A to D: Fixator-assisted nailing technique: (A) Preoperative anteroposterior femur radiograph demonstrating a valgus deformity with a mechanical lateral distal femoral angle (mLDFA) measuring $76^{\circ}$; (B) Intraoperative image demonstrating a half pin in the distal fragment holding the correction via a pin-to-bar construct (proximal half pin not visible). Placement of the pin close to the osteotomy helps to give better manipulative control of the segment; (C) Intraoperative lateral image of the distal femur demonstrating the position of the half-pin posterior to the potential path of the nail; (D) Anteroposterior femoral radiograph demonstrating the final result after a $3.5 \mathrm{~cm}$ lengthening. Patient has a normal mLDFA. Note the blocking screw placed lateral to the nail in the distal fragment

\section{Reverse Planning Method (Fig. 3)}

Preoperative surgical planning was performed using Paley's principles of deformity analysis and Baumgart's reverse planning method. ${ }^{17,20}$ The level of the osteotomy, the nail length and the nail diameter were also determined preoperatively. The digital radiographic images were loaded into the Bone Ninja App (International Center for Limb Lengthening, Baltimore, Maryland, USA) to illustrate the deformity correction. ${ }^{21}$ Using the Bone Ninja App, the amount of angulation and translation of the distal femoral fragment to restore a neutral mechanical axis could be visualised. The orientation of the intramedullary nail within each fragment could also be illustrated. These images were printed and brought to the operating room to guide the surgeon. The level of the osteotomy and the proximal extent of reaming were marked on the patient under fluoroscopic guidance. Venting of the osteotomy site was performed by placing multiple percutaneous drill holes. A medial parapatellar approach to the knee was made. Based on the preoperative plan, the nail guide wire was then inserted aiming medially towards the osteotomy drill holes. A rigid reamer was used to ream over the guide wire up to the osteotomy site. A blocking screw(s) was placed lateral to the rigid reamer to hold its path. The percutaneous osteotomy was then completed using an osteotome. The required correction was performed by rotating, translating and angulating the distal fragment with the rigid reamer in place. Once the desired correction was achieved, the reamer was advanced into the proximal segment. Additional blocking screws were applied around the reamer as necessary to maintain the alignment. The reamer was then exchanged for the nail. The nail was locked proximally and distally. Restoration of the mechanical axis was verified intraoperatively using a grid.

The mLDFA was measured preoperatively and postoperatively for all patients using femoral radiographs. All the measurements were performed by two surgeons independently. Data regarding the range of motion ( $\mathrm{ROM})$, operative time, complications
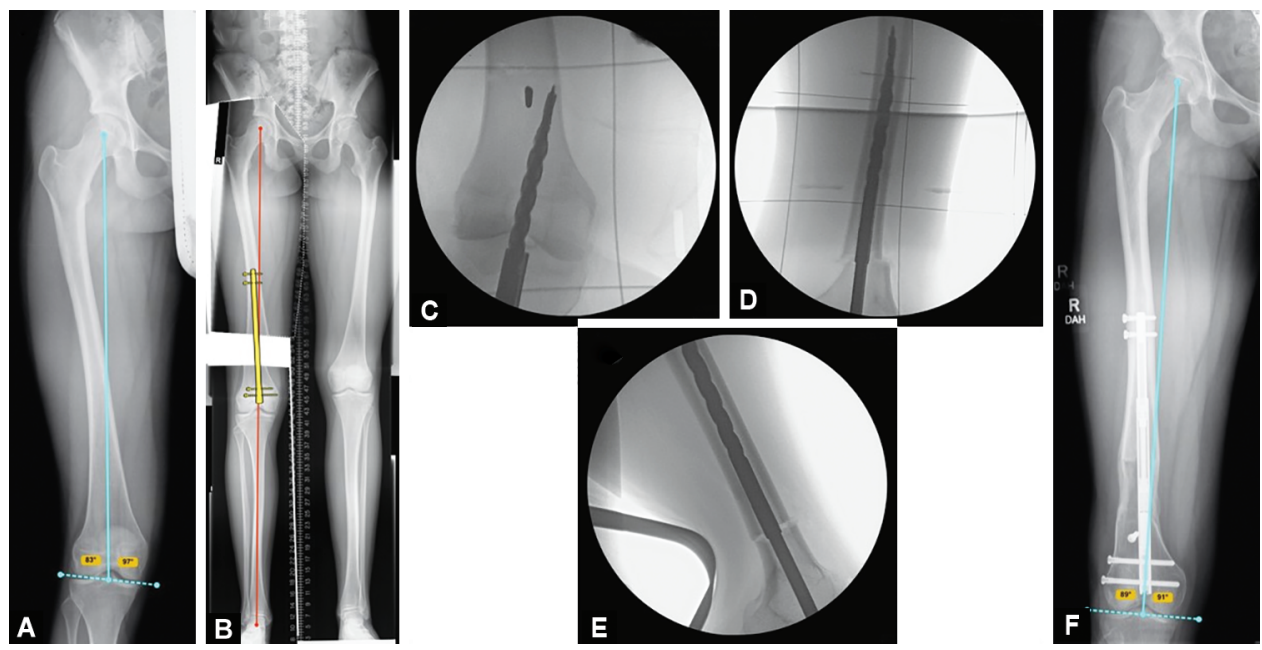

Figs 3A to F: Reverse planning technique: (A) Preoperative anteroposterior femur radiograph demonstrating a valgus deformity with a mechanical lateral distal femoral angle (mLDFA) measuring $83^{\circ}$; (B) Illustration of final alignment with retrograde nail in place using the reverse planning method; (C) Intraoperative image demonstrating the guide wire with cannulated rigid reamer angled medially towards the proposed osteotomy site (drill holes) per the preoperative plan. A blocking element has been placed to prevent the reamer from moving out of this position during the acute correction step; (D) Intraoperative image with the rigid reamer passed into the proximal segment; (E) Intraoperative lateral image demonstrating the rigid reamer passed into the proximal segment. It is important to monitor the reaming in the lateral view to avoid reaming the anterior cortex of the femur; (F) Anteroposterior femoral radiograph demonstrating the final result after a $5 \mathrm{~cm}$ lengthening. Patient has a normal mLDFA. Note the blocking screw placed lateral to the nail in the distal fragment 
Table 1: Summary statistics

\begin{tabular}{lllll}
\hline & $\begin{array}{l}\text { Reverse planning } \\
(n=10)^{*}\end{array}$ & FAN $(n=11)$ & FAP $(n=6)^{* *}$ & $p$ value \\
\hline Age & $15.7 \pm 2.2$ years & $16.4 \pm 5$ years & $15 \pm 2.4$ years & $12-17$ years \\
Age range & $12-18$ years & $12-31$ years & $82 \pm 1.1^{\circ}$ & 0.2897 \\
mLDFA preoperative & $80.6 \pm 3.7^{\circ}$ & $79.5 \pm 2.9^{\circ}$ & $80-83^{\circ}$ & 0.3440 \\
mLDFA range preoperative & $75-86^{\circ}$ & $75-83^{\circ}$ & $88 \pm 1.7^{\circ}$ & 0.5832 \\
mLDFA postoperative & $88.3 \pm 1.8^{\circ}$ & $87.3 \pm 1.3^{\circ}$ & $86-90^{\circ}$ & 0.0006 \\
mLDFA range postoperative & $86-92^{\circ}$ & $86-90^{\circ}$ & $28.7 \pm 3.2$ & 0.8190 \\
BMI & $34.4 \pm 19.8$ & $28.9 \pm 8.4$ & $110.7 \pm 12.7$ minutes \\
Duration of surgery & $113.2 \pm 26.6$ minutes & $150.6 \pm 20$ minutes & $115 \pm 8.4^{\circ}$ & $539.8 \pm 165.6$ \\
Range of motion & $117.4 \pm 14.6^{\circ}$ & $119.8 \pm 18^{\circ}$ & & \\
Follow-up duration (in days) & $194.4 \pm 84.9$ & $451.7 \pm 244.2$ & & \\
\hline
\end{tabular}

${ }^{*} n=10$ limbs in 9 patients

${ }^{* *} n=6$ limbs in 4 patients

FAN, fixator-assisted nailing; FAP, fixator-assisted plating; mLDFA, mechanical lateral distal femoral angle; BMI, body mass index

associated with the procedure and body mass index (BMI) were collected from the medical charts.

GraphPad Prism (version 8.2.0 GraphPad software Inc., San Diego, California, USA) statistical package was used for analysis. The student's t-test and single-factor analysis of variance (ANOVA) with Tukey's post hoc test were used when indicated, to compare means with the significance level set at a confidence level of $95 \%$ and $p$ value $<0.05$.

\section{Results}

Twenty-seven limbs in 24 skeletally mature patients (8 males, 16 females) with distal femoral valgus deformities underwent correction. Ten limbs in 9 patients had correction using the reverse planning method, 11 limbs in 11 patients had FAN while 6 limbs in 4 patients had FAP. In the reverse planning group, six limbs underwent correction only, while four had correction and lengthening. In the FAN group, 10 patients had correction and lengthening, while 1 had correction only.

The mean preoperative and postoperative mLDFA in the reverse planning group was $80.6^{\circ}$ and $88.3^{\circ}(p<0.0001)$. In the FAN group, the values were $79.5^{\circ}$ and $87.3^{\circ}(p<0.0001)$ while in the FAP group, the values were $82^{\circ}$ and $88^{\circ}(p<0.0001)$ (Table 1$)$. All patients had restoration of the mLDFA to within normal range of $85-90^{\circ}$ except for one patient in the reverse planning group who had a postoperative angle of $92^{\circ}$. This overcorrection was performed purposefully in order to compensate for a slightly valgus tibia in this patient. There was no statistically significant difference in the preoperative or final mLDFAs between the groups (preoperative and postoperative $p$ values $=0.2897$ and 0.3440 , respectively). We found a statistically significant difference in surgical time between the reverse planning and the FAN group $(p=0.0016)$, as well as the FAN and the FAP group $(p=0.0035)$. There was no statistical difference in operating time between the reverse planning group and the FAP group ( $p=0.9720$ ).

The mean final knee ROM in the reverse planning group, the FAN group and the FAP group were $117.4 \pm 14.6^{\circ}, 119.8 \pm 18^{\circ}$ and $115 \pm 8.4^{\circ}$, respectively. There was no statistically significant difference in the ROMs across the groups $(p=0.8190)$. Although the reverse planning group had the highest average BMI, this was not statistically significantly different than the average BMI in the FAN or the FAP groups.

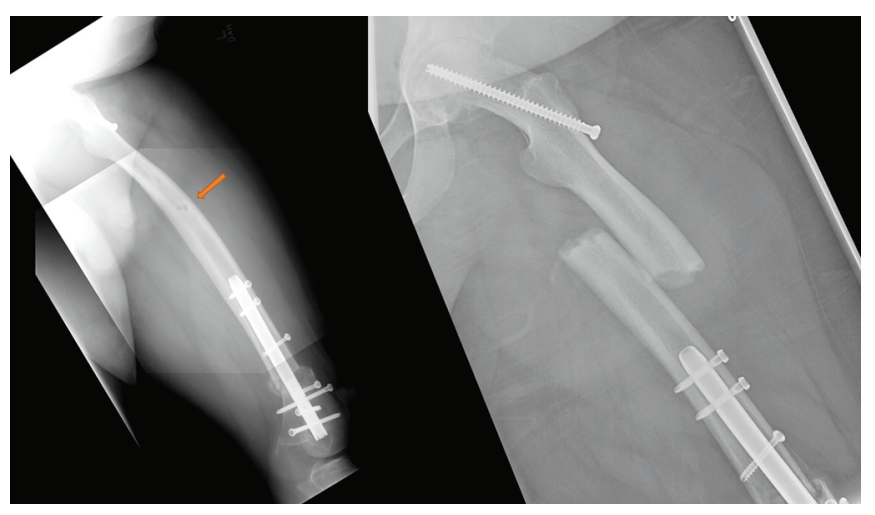

Fig. 4: (Left) Lateral femoral radiograph indicating the location of a bicortical diaphyseal pin site hole (red arrow) after a fixator-assisted nailing to correct femoral valgus deformity. Note that the pin site hole is in the centre of the bone; (Right) Lateral radiograph demonstrating a fracture through the pin site hole after the patient slipped on a wet floor at home

There were no postoperative infections in any group. There were no delayed unions or non-unions in any of the groups. One patient in the reverse planning group had irritation around the distal screws laterally, but the knee pain caused no restriction in activities and did not require any pain medications. One patient in the FAN group suffered a fracture through the proximal half-pin site at 6 weeks after surgery when he slipped on a wet floor (Fig. 4). This required a temporary external fixator and then conversion to a longer retrograde nail. His osteotomy and periprosthetic fracture healed without loss of alignment. In the FAP group, all six plates were eventually removed after union per patient request due to localised discomfort from the hardware.

\section{Discussion}

Valgus deformity in the distal femur can occur in patients with a congenital limb deficiency, such as fibular deficiency, or it can be acquired due to a traumatic, metabolic, infectious or neoplastic process. Unaddressed distal femur valgus deformity can lead to pain, decreased function and secondary arthritic changes in the knee. $^{2}$ In skeletally immature patients, guided growth can be used to correct the femoral alignment gradually. ${ }^{22}$ However, in skeletally 
mature patients, a distal femoral osteotomy is required to restore normal alignment.

This study reviews three different methods for correcting distal femoral valgus via an osteotomy in skeletally mature patients: FAP, FAN and reverse planning. Both the FAN and the FAP techniques are based on the concept that the desired correction is obtained intraoperatively using an external fixator but maintained postoperatively by the internal hardware. ${ }^{5-16}$ This allows the surgeon to take advantage of the best feature of each modality (the adjustability of an external fixator and the patient convenience of the internal hardware) while avoiding the disadvantages (external fixator as definitive management, and difficulty adjusting the exact amount of correction with internal fixation). ${ }^{9}$ The reverse planning method avoids the intraoperative fixator by relying on meticulous preoperative planning, the use of rigid reamers and the judicious use of blocking screws to guide and maintain the correction. ${ }^{17}$

Previous studies have demonstrated the effectiveness of each technique in achieving deformity correction. Eidelman et al. showed in their series of six patients that minimally invasive FAP is a reliable and accurate method in correcting distal femur valgus deformity. ${ }^{6}$ Eralp et al. retrospectively reviewed 17 patients who underwent knee deformity correction procedures using the FAN and the circular external fixator techniques. ${ }^{12}$ Nine patients were treated with the FAN technique, and eight patients were treated with the circular external fixator technique. They demonstrated that the accuracy of correction using the FAN was comparable to the external fixation technique. Fragomen and Rozbruch and lobst et al. also demonstrated retrograde femoral magnetic intramedullary lengthening nails could be used to correct distal femur deformity and leg length discrepancy at the same time. ${ }^{13,14}$ Baumgart originally described accurate deformity correction with or without lengthening using the reverse planning method. ${ }^{17}$ This has been corroborated by authors from Turkey and Switzerland who used the reverse planning method to correct femoral deformity in combination with intramedullary limb lengthening. ${ }^{18,19}$

Two studies have directly compared the results of FAN and FAP. ${ }^{15,16}$ Kovar et al. conducted a retrospective review of 24 patients with distal femoral valgus deformity with 18 patients (20 femurs) in the FAN group and 6 patients (7 femurs) in the FAP group. ${ }^{16}$ The study demonstrated no statistically significant difference in the accuracy of correction between the two groups. Galal conducted a retrospective review of 18 patients who underwent distal femoral osteotomy using the FAN in 12 patients and the FAP in 6 patients. ${ }^{15}$ They also found no statistically significant difference in the accuracy of correction with either technique.

Our study is the first to compare all three techniques of distal femoral realignment osteotomy in a single series of patients with comparable deformity (distal femoral valgus). We found that each method can accurately restore alignment. There was no statistically significant difference between the groups in terms of the final mLDFA. Only one patient from the entire series had a final mLDFA outside the desired range of $85-90^{\circ}$. This patient came from the reverse planning group and was purposely overcorrected by $2^{\circ}$ to compensate for ipsilateral tibial valgus.

In this study, the FAN technique had the longest average surgical time which was statistically significant compared to the FAP and the reverse planning groups. This may be related to several factors. The reverse planning cases do not require the extra time necessary to place and manipulate the external fixator as in the FAN patients. The FAP cases did not need extra time to insert blocking screws like the FAN procedures and it is easier to place the fixator pins since they do not have to precisely place to avoid the canal space. In addition, placement of screws in the plate usually takes less time than placing proximal and distal interlocking screws in the nail. While the FAP and the reverse planning methods had comparable operative times, only the reverse planning method allows the option to perform both deformity correction and lengthening.

In terms of the outcome, there were no infections, delayed unions or non-unions in any group. There was also no statistically significant difference in final knee ROM between the groups which is in line with previous studies. ${ }^{5-16}$ Additional surgeries, however, were required in patients from the FAN and the FAP groups. One patient from the FAN group suffered a fracture through a proximal half-pin site when he slipped on a wet floor in his home. Since he was only 6 weeks from surgery and the osteotomy was not fully healed, he underwent temporary external fixation of the periprosthetic fracture. A longer retrograde nail that spanned the osteotomy and the fracture site was inserted a few weeks later when the osteotomy was fully healed. Analysis of the half-pin hole fracture site demonstrated that it had been placed in the centre of the bone (not unicortical). However, it was located in the femoral diaphysis and not at the level of the less trochanter, which may have increased its potential to be a stress riser. All six limbs (four patients) with FAP gradually developed discomfort around the hardware after union and requested hardware removal. Each procedure was performed as outpatient surgery and did not cause any additional complications. The request for plate removal is a consistent finding that has been noted in all of the FAP literature. ${ }^{5-8}$ Although the plates are precontoured to fit the shape of the lateral distal femur, the proximal portion of the plate must sit off the bone to maintain the desired correction. This plate position, plus the relatively large size of the plate (4.5 $\mathrm{mm}$ ), adds bulk to the lateral thigh along the iliotibial band that may become uncomfortable once the patient has returned to full activities. One patient in the reverse planning group mentioned having lateral knee discomfort from an interlocking screw but did not require any additional treatment. This finding is likely related to the placement of the hardware itself and is independent of the surgical technique.

This study has several limitations. It is a retrospective review of a single surgeon's experience. Although the nail patients were performed consecutively (initially all FAN and then all reverse planning), a randomised grouping would have been a more valuable research design. Although our sample size was comparable to previously published studies, the total number of patients is relatively small, with 10 patients in the reverse planning group, 11 in the FAN and 6 in the FAP group. Future studies with larger patient numbers in each group need to be conducted to confirm our findings. No patient reported outcome measures were obtained on these patients which also would have added value of the findings of this study. In the two nail groups (FAN and reverse planning), the percentage of patients undergoing lengthening and deformity correction were not identical (40\% reverse planning vs $91 \%$ FAN). However, both groups had an accurate final correction and there were no delays in healing. Finally, because the nail patients had various amounts of lengthening combined with the deformity correction, we were unable to provide a uniform calculation of the time to union and the time to full weight-bearing. Therefore, this information was not included in this study. We can report that there were no delays in healing in any of the patients. 


\section{Conclusion}

All three methods of deformity correction, FAN, FAP and reverse planning, accurately correct distal femoral valgus deformity in skeletally mature patients. Each method also results in similar final knee ROM and union rates. However, patients in the FAN and the FAP groups required additional surgeries not seen in the reverse planning group. In addition, the FAP and the reverse planning methods had statistically significantly shorter operative times comparted to the FAN method. While the reverse planning technique may require a slightly more complicated preoperative planning process, it had the best combination of surgical outcomes in this study. It was faster than the FAN technique, allowed for both deformity correction and lengthening and avoided the additional surgeries seen in the FAP and the FAN patients. Ultimately, however, the decision of which technique to use depends on a combination of the patient's preference and the surgeon's level of comfort with the technique.

\section{References}

1. Sharma L, Song J, Felson DT, et al. The role of knee alignment in disease progression and functional decline in knee osteoarthritis. JAMA 2001;286(2):188-195. DOI: 10.1001/jama.286.2.188.

2. Sculco PK, Kahlenberg CA, Fragomen AT, et al. Management of extraarticular deformity in the setting of total knee arthroplasty. J Am Acad Orthop Surg 2019;27(18):e819-e830. DOI: 10.5435/JAAOS-D-18-00361.

3. Marangoz S, Feldman DS, Sala DA, et al. Femoral deformity correction in children and young adults using Taylor spatial frame. Clin Orthop Rel Res 2008;466(12):3018-3024. DOI: 10.1007/s11999-008-0490-2.

4. Want JW, Hsu CC. Distal femoral varus osteotomy for osteoarthritis of the knee. J Bone Joint Surg Am 2005;87(1):127-133. DOI: 10.2106/ JBJS.C.01559.

5. Seah KT, Shafi R, Fragomen AT, et al. Distal femoral osteotomy: is internal fixation better than external? Clin Orthop Relat Res 2011;469(7):2003-2011. DOI: 10.1007/s11999-010-1755-0.

6. Eidelman M, Keren $Y$, Norman D. Correction of distal femoral valgus deformities in adolescents and young adults using minimally invasive fixator-assisted locking plating (FALP). J Pediatr Orthop B 2012;21(6):558-562. DOI: 10.1097/BPB.0b013e328358f884.

7. Rozbruch SR. Fixator-assisted plating of limb deformities. Oper Tech Orthop 2011;21(2):174-179. DOI: 10.1053/j.oto.2011.01.005.

8. Yilmaz G, Bakircioglu S. Correction of distal femoral valgus deformities with fixator-assisted plating: how accurate is the correction? Acta Orthop Traumatol Turc 2019;53(2):100-105. DOI: 10.1016/j. aott.2018.11.002.
9. Paley $D$, Herzenberg JE, Bor N. Fixator-assisted nailing of femoral and tibial deformities. Tech Orthop 1997;12(4):260-275. DOI: 10.1097/00013611-199712000-00004.

10. Kocaoglu M, Bilen FE. Fixator-assisted nailing for correction of long bone deformities. Operat Tech Orthop 2011;21(2):163-173. DOI: 10.1053/j.oto.2011.01.010.

11. Kocaoglu M, Eralp L, Bilen FE, et al. Fixator-assisted acute femoral deformity correction and consecutive lengthening over an intramedullary nail. J Bone Joint Surg Am 2009;91(1):152-159. DOI: 10.2106/JBJS.H.00114.

12. Eralp L, Kocaoglu M, Toker B, et al. Comparison of fixator-assisted nailing versus circular external fixator for bone realignment of lower extremity in rickets. Arch Orthop Trauma Surg 2011;131(5):581-589. DOI: 10.1007/s00402-010-1162-8.

13. Fragomen AT, Rozbruch SR. Retrograde magnetic internal lengthening nail for acute femoral deformity correction and gradual limb lengthening. Expert Rev Med Devices 2017;14(10):811-820. DOI: 10.1080/17434440.2017.1378092.

14. Iobst CA, Rozbruck SR, Nelson S, et al. Simultaneous acute femoral deformity correction and gradual lim lengthening using a retrograde femoral nail: Technique and clinical results. J Am Acad Orthop Surg 2018;26(7):241-250. DOI: 10.5435/JAAOS-D-16-00573.

15. Galal S. Comparison of fixator-assisted plating versus fixator-assisted nailing for distal femoral osteotomy. J Limb Lengthening Reconstr 2017;3(1):52-56. DOI: 10.4103/jllr.jllr_25_16.

16. Kovar FM, Jauregui JJ, Herzenberg JE. Accuracy of distal femoral valgus deformity correction: fixator-assisted nailing versus fixatorassisted locked plating. Am J Orthop 2018;47(6). DOI: 10.12788/ ajo.2018.0044.

17. Baumgart $R$. The reverse planning method for lengthening of the lower limb using a straight intramedullary nail with or without deformity correction. Oper Orthop Traumatol 2009;21(2):221-233. DOI: 10.1007/s00064-009-1709-4.

18. Kucukkaya M, SokucuS, Thaller PH. Surgical techniques for lengthening and deformity correction of the femur with lengthening nails. Tech Orthop 2015;30(3):183-188. DOI: 10.1097/BTO.0000000000000137.

19. Lenze $\mathrm{U}$, Krieg AH. Intramedullary lengthening nails: Can we also correct deformities? J Child Orthop 2016;10(6):511-516. DOI: 10.1007/ s11832-016-0782-0.

20. Paley D. Normal lower limb alignment and joint orientation. In: Principles of Deformity Correction 2002. 1-18.

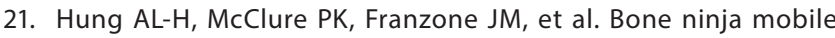
app for reverse planning method in internal limb deformity and lengthening surgery. Strategies in Trauma and Limb Reconstruction 2019;14(2):72-76. DOI: 10.5005/jp-journals-10080-1425.

22. Kearney SP, Mosca VS. Selective hemiepiphyseodesis for patellar instability with associated genu valgum. J Orthop 2015;12(1):17-22. DOI: 10.1016/j.jor.2015.01.005. 